\title{
COMMUNICATIONS
}

\section{OCULAR CHANGES IN ACUTE SYSTEMIC LUPUS ERYTHEMATOSUS*}

\author{
BY
}

\author{
F. CLIFTON AND C. H. GREER
}

From the Bristol Eye Hospital, and the Department of Pathology, Institute of Ophthalmology, University of London

Although ocular lesions are known to occur in acute systemic lupus erythematosus, the only report in Great Britain of the histological examination of the eyes in this disease appears to be that of Semon and Wolff (1933), who found only mild choroiditis and subretinal exudation. The purpose of this paper is to record the clinical, ophthalmoscopic, and pathological findings in two cases which terminated fatally.

\section{Case Reports}

Case 1, a married woman, aged 24, a diabetic of 8 years' standing, attended the Dermatological Department of the Bristol General Hospital in June, 1949, complaining of an irritating red rash of 2 months' duration on her forehead, face, and ears.

Examination.-A scaling erythematous rash was present on the forehead extending down to the bridge of the nose and on to both cheeks and both ears. Her temperature was $99 \cdot 2^{\circ} \mathrm{F}$. General physical examination revealed only a systolic murmur heard in the mitral area. She was admitted under the care of Dr. C. D. Evans.

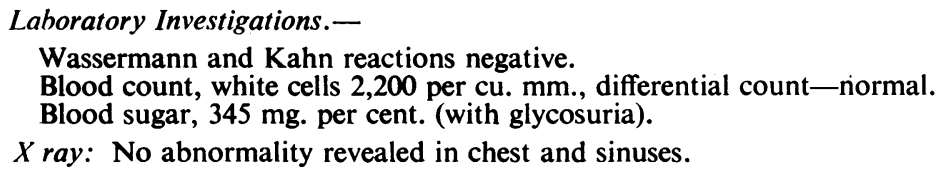

Course and Therapy.-She was treated by rest in bed, bland local applications, and an increase in insulin. On discharge 5 weeks later, the rash was much paler and less extensive. She then attended as an out-patient, but was readmitted in September with a diagnosis of acute systemic lupus erythematosus. The rash now covered the whole of the face, trunk, and limbs, but was more severe and darker red on the face, ears, and dorsa of the hands and feet. The extensor muscles were tender on pressure, and there was generalized stiffness, pain, and muscular weakness. A muscle biopsy showed only roundcell and plasma-cell interstitial infiltration without loss of muscle striation, so that dermatomyositis was ruled out. A general physical examination revealed no other abnormalities. The diabetes was controlled.

From the time of readmission, her condition gradually deteriorated. Irregular pyrexia $\left(99^{\circ}-101^{\circ} \mathrm{F}\right.$.) persisted, in spite of aspirin and a 5 -day course of procaine penicillin, 300,000 units morning and evening.

By December a severe diffuse alopoecia of the scalp was present. A normochromic anaemia was treated by a transfusion of $1 \frac{1}{2}$ pints of blood. On December 16 a small 
pericardial effusion developed and the spleen became palpable. Meanwhile the rash was fading. On January 19, during a 5-day course of aureomycin, she suffered a short epileptiform attack involving the arms and head.

On January 23, examination of the eyes revealed similar changes in both fundi (Fig. 1). There was slight but definite congestion of the retinal veins and a circumpapillary oedema, most pronounced around the upper margin of the discs which were not swollen. In the region of the posterior poles there were several discrete superficial lesions, the larger appearing like cotton-wool patches, the smaller of slightly firmer texture with a faintly pinkish tint. These were situated in the course of, or adjacent to, both arteries and veins. Except for a slightly heightened reflex, the arteries appeared normal. Scattered superficial haemorrhages were noted in the vicinity of vessels and there were one or two punctate lesions which were thought to be micro-aneurysms. Uncorrected visual acuity was recorded as $6 / 9$ in both eyes.

Fig. 1.-Case 1, fundus drawing of left eye 6 weeks before death, showing circumpapillary oedema, venous congestion, aggregations of cytoid bodies, and superficial haemorrhages.

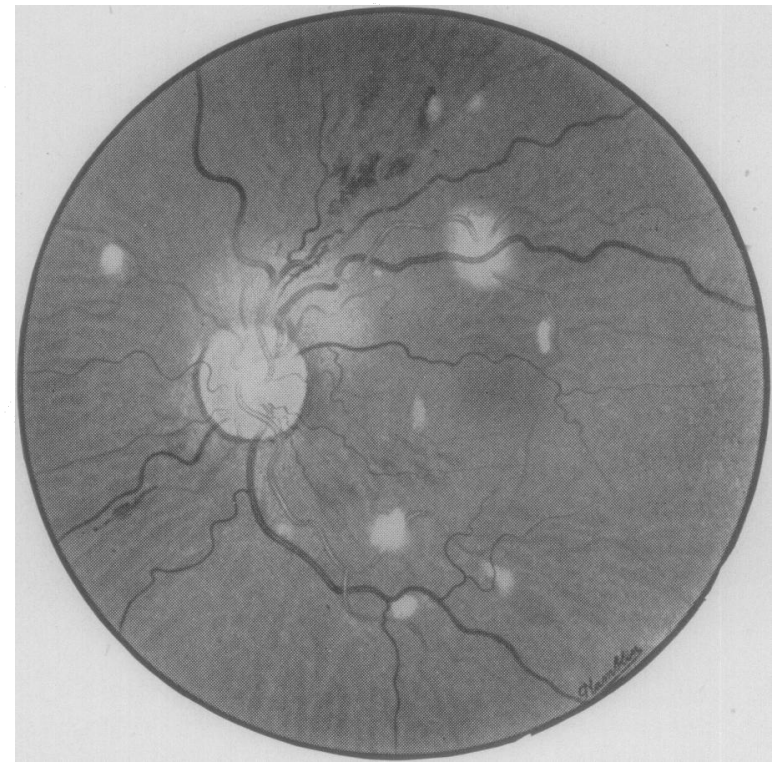

By February 7, the patient was critically ill, with temperature $102^{\circ} \mathrm{F}$., severe retrosternal pain which was worse on swallowing, and a palpable tender spleen. Radiography showed considerable widening of the cardiac shadow, and pleural effusion at both lung bases. An injection of $25 \mathrm{mg}$. ACTH produced rapid marked improvement. Within 2 hours the patient became cheerful and talkative and her appetite returned. After 4 hours she could move her arms freely and without pain. Before the injection of ACTH the urine had been free of sugar and acetone, but 24 hours later, these reappeared and the dose of insulin had to be increased. Administration of ACTH was continued in 25-mg. doses at 6-hrly intervals until February 11, the patient's improvement being maintained.

From February 11 to 15 a similar dose was given at 12-hrly intervals, but the patient's general condition deteriorated slightly.

From February 15 to $27,25 \mathrm{mg}$. ACTH were given daily. During this period, the skin lesions disappeared, and fundus examination on the 12th day after the commencement of ACTH therapy revealed a reduction in the size of the larger white exudative patches and a diminution of venous congestion. The superficial haemorrhages persisted. ACTH therapy was discontinued on February 27 for 3 days, as the patient had developed a urinary infection with $B$. coli and Ps. pyocyanaeus. This was treated by injections of $1 \cdot 5 \mathrm{~g}$. streptomycin daily in divided doses. 
In spite of the re-institution of ACTH therapy at 8-hrly intervals from March 2, the patient's condition steadily deteriorated. She took very little by mouth, muscular weakness returned, and there was increasing anæmia, only slightly and temporarily improved by a transfusion of two pints of blood (packed cells). She remained mentally clear and coherent until she died on March 11.

Post-Mortem Examination.--A very emaciated young adult, prematurely aged, with very little hair on the scalp. There was some scaling of the skin but no lesions of lupus erythematosus remained. The lungs were small and congested, being compressed by a large distended pericardium; the pleural cavities were obliterated by recent adhesions, the pericardium was greatly distended by $1 \frac{1}{2}$ pints of haemorrhagic exudate with mucoid brownish pus forming a thick sediment. The epicardium had shaggy brownish exudate adherent to it. The heart was small and contracted. There were no valvular lesions. The suprarenal glands were deficient in cortical lipoid but the medulla was large and bulky. No abnormalities were found in any of the other organs except some fatty change in the liver.

Histological Examination.-The pericardium showed a serofibrinous exudate organizing in places, and the surface layer showed more recent purulent infiltration. Sections of the lungs showed a terminal oedema, fatty infiltration was present in the liver, and cloudy swelling in the kidneys. No gross abnormality was seen in the cardiac muscle, pancreas, spleen, aorta, and pituitary or suprarenal glands.

Ocular Findings. - The left eye was removed post mortem, fixed in 10 per cent. formalin, and opened by para-saggital section. One portion was embedded in celloidin and sections stained with haematoxylin and eosin. The other portion was embedded in carbo-wax and the sections from it stained with Scharlach R.

Macroscopically scattered exudates and one or two retinal micro-aneurysms were visible.

Microscopically the main pathological changes were in the retina. Its fibre layer showed diffuse and marked oedema with scattered aggregations of cytoid bodies, frequently but not invariably associated with superficial haemorrhages. Oedema was present in both plexiform layers and the retinal vessels were congested. The layer of rods and cones had been almost completely transformed into granular subretinal fluid resulting in shallow detachment. Scattered round cells were present in the choroid. The anterior segment of the eye and the optic nerve were normal in appearance.

Case 2, a female, aged 18 years, was admitted to the Bristol Royal Infirmary under the care of Dr. A. M. G. Campbell on October 18, 1952, with a diagnosis of acute disseminated lupus erythematosus. In the previous June she had begun to lose her hair and towards the end of August had started to run a temperature accompanied by muscular pains and tenderness in her thighs and shoulders. She had also noticed some redness of her face. After being ill for a month she started to vomit and soon after was admitted to a fever hospital as a suspected case of paratyphoid fever. There she was found to present a typical picture of acute systemic lupus erythematosus, with alopoecia, facial rash of " butterfly" distribution, and haemorrhagic spots on the pulps of her fingers. A small subconjunctival haemorrhage was present in the left eye; both fundi were normal. Her urine contained albumen and casts. The spleen and glands were not enlarged and no abnormal signs were noted in the heart or lungs. She had been treated by large doses of aspirin, with a resulting fall in temperature and improvement in symptoms, but this had been discontinued after a few days because of vomiting.

Examination.-A pale, lethargic young woman with marked alopoecia of the scalp. She complained of stiffness and pain on movement of hips and knees. Her pulse was 92 and regular, and blood pressure 125/85. The rash, which had largely faded from her face, was now evident on the extensor surfaces of both arms. Her teeth were in good condition, but there was bleeding from the gums. The axillary glands were enlarged. No abnormal signs were noted in the chest, abdomen, or central nervous system. 


\section{Laboratory Investigations.-}

Wassermann and Kahn reactions negative.

E.S.R. (Wintrobe) $55 \mathrm{~mm}$. in $1 \mathrm{hr}$.

Blood count showed iron deficiency anaemia (Hb. 50 per cent.).

White cell count, 3,000 per cu. $\mathrm{mm}$. with normal differential.

Blood urea, $36 \mathrm{mg}$. per cent.

Urine contained albumen, blood, and granular casts, scanty pus cells but no organisms; culture was sterile.

$\mathrm{X}$ ray of chest showed no evidence of a gross active lesion of the lungs. There was a calcified primary complex in the right upper zone.

The L.E. phenomenon was demonstrated using the patient's plasma and leucocytes and the plasma and leucocytes from another person.

Course and Therapy.-On October 24 the patient, who appeared very ill, was complaining of mistiness of vision. Externally the eyes appeared normal. Both fundi (Fig. 2) showed numerous retinal lesions of cotton-wool like appearance, mainly situated around the disc, which, as in Case 1, was not swollen, though there was circumpapillary oedema. These lesions were mostly discrete and in proximity to arteries and veins, but a large aggregate of slightly firmer texture was situated between the disc and the macula in each eye. The central retinal veins were congested. Only a few small haemorrhages were noted in either eye. Two are to be seen in Fig. 2, one ensheathing a small segment of the superior temporal vein at a point about a disc diameter from the nerve head, and the other fringing the upper margin of an exudative patch below the macula. The upper and lower divisions of the central retinal artery showed an increased reflex which did not extend beyond the area of circumpapillary oedema.

Fig. 2.-Case 2, fundus drawing of right eye 4 days before death, showing circumpapillary oedema, venous congestion, superficial haemorrhages, and numerous aggregations of cytoid bodies.

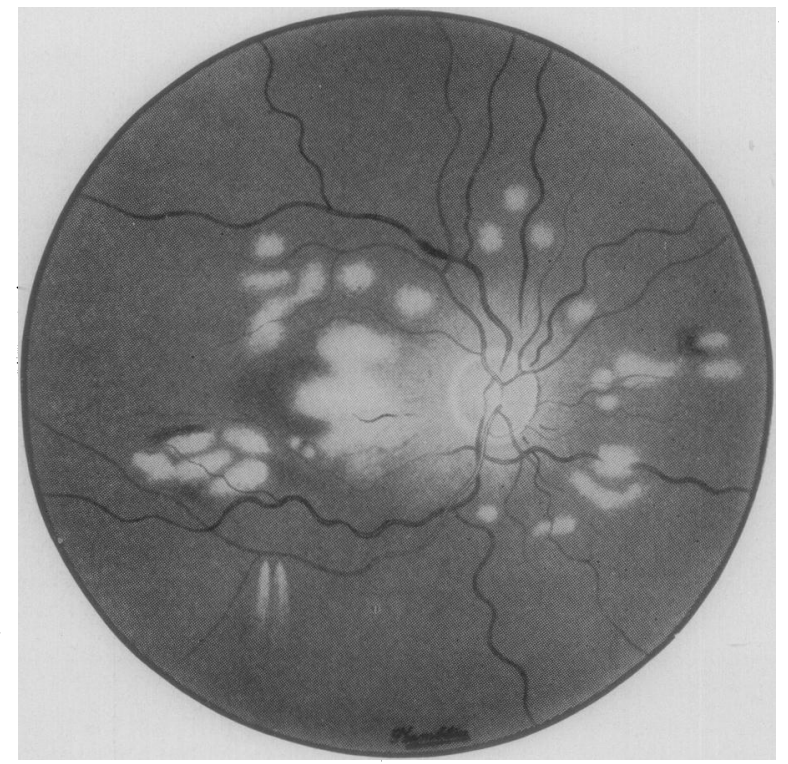

From the time of this examination the patient's condition deteriorated rapidly despite treatment with mepacrin $100 \mathrm{mg}$. twice daily and penicillin. Terminally, the blood urea rose to $150 \mathrm{mg}$. per cent. and there was an increase in the white cell count. She developed signs of consolidation first at the base of the left lung and then more generally throughout both lungs. Her pulse increased to $\mathbf{1 4 0}$ with a marked gallop rhythm, her temperature rose to $105^{\circ} \mathrm{F}$., cyanosis, and dyspnoea developed, and death occurred on October 28 , 10 days after admission. 
Post-Mortem Examination.-A well-nourished, well-developed woman. There was residual scabbing at the sides of the nose, but no evidence of a " butterfly" rash; petechial haemorrhages in the left forearm, wrists, and elbow flexures; slight freckling of neck, face, and forearms; no other rash seen. The lungs showed considerable rather solid oedema; there was no broncho-pneumonic consolidation, but the whole of the parenchyma was congested and felt distinctly firm. There were multiple small haemorrhages of lobular distribution. The heart was slightly dilated but not hypertrophied, the myocardium being soft and pale with slight evidence of fatty change. No haemorrhages, valvular lesions, or thrombi were seen. Both pericardial and pleural cavities contained a clear yellow fluid. Positive findings in other organs were as follows:

Spleen: acutely swollen, one or two tiny pyaemic abscesses present.

Liver: severe fatty change.

Lymph nodes: enlarged, congested and oedematous in all parts of the body; calcified lymph node above right hilum.

Adrenals: complete loss of lipid in thin cortex.

Kidneys: acutely swollen, with pale cortex and red medulla; a few scattered pyaemic abscesses were noted.

Right middle ear purulent inflammation; sphenoidal sinus full of pus.

Histological Examination.-Of the findings in the various organs the most striking were those in the kidneys. "Wire-looping" of the glomerular capillaries was present. A few loops contained hyaline bodies which were generally eosinophilic but sometimes basophilic as well. Occasionally they were stippled with basophilia, as though containing nucleic fragments, possibly representing the haematoxophilic bodies of Gross. In places there were small patches of tubular atrophy with epithelial dissociation which might have been due to ischaemia. The blood vessels appeared normal; no real tuft infarction was present, though occasional slight adhesions were noted. The spleen and axillary lymph nodes showed reactive hyperplasia. The lungs showed patchy alveolar haemorrhages in various stages of organization, but none more than 2 or 3 weeks old. There were also a few small areas of acute inflammatory cellular infiltration, possibly pyaemic. The femoral marrow showed a well-marked reactive hyperplasia in which L.E. cells were absent. A few perivascular round cells were noted in the psoas muscle. In the pectoral, there were also lymphoid aggregates. In the skin petechial haemorrhages were present in the papillary dermis, but there was no arteriolitis. The overlying keratin showed evidence of previous vesicular eruption.

Ocular Findings.-The right eye was removed post mortem, fixed in 10 per cent. formalin, and opened by para-saggital section. The larger portion was embedded in celloidin and sections stained with haematoxylin and eosin. The smaller portion was embedded in paraffin wax and sections from it stained with Mallory's phosphotungstic acid haematoxylin and with Holmes's silver stain.

Macroscopically the retina appeared grey and opaque. Round the optic disc it was raised into a mound, upon the surface of which were several haemorrhages and numerous superficial cotton wool spots.

Microscopically the principal changes were seen in the retina which was thick and oedematous, especially posteriorly around the disc where large aggregations of cytoid bodies distended the nerve fibre layer and distorted the cellular layers (Fig. 3, overleaf). Among the cytoid bodies spherical hyaline globules of smaller size were evident in considerable numbers. Large accumulations of albuminous transudate with hyaline centres were present in the outer plexiform layer. Over a wide area the nerve fibres were necrotic but this was probably post-mortem degeneration. Near the disc the rods and cones had undergone granular degeneration inducing a shallow retinal detachment. Scattered lymphocytes and scanty Russell bodies were present in the choroid. There was evidence of mild iridocyclitis and the lens showed anterior cortical vacuolation. The cornea and sclera were normal. 
FIG. 3.-Photomicrograph of retina (Case 2) showing large aggregation of cytoid bodies distending nerve fibre layer. Haematoxylin and eosin $\times 120$.

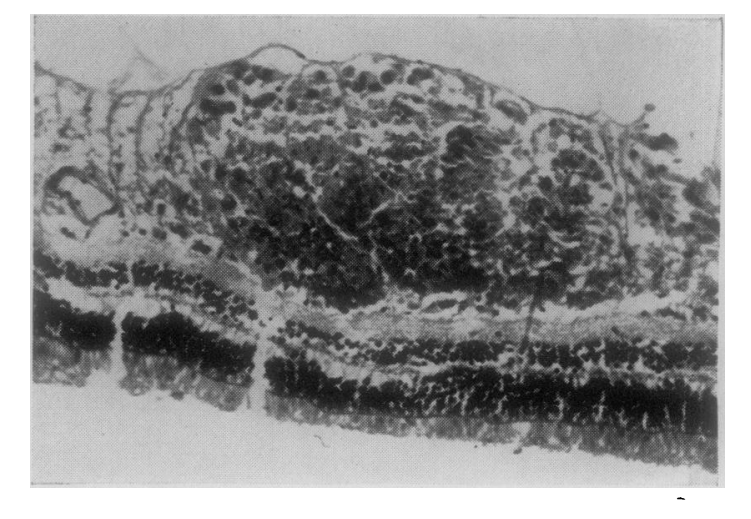

\section{Discussion}

Lupus erythematosus manifests itself either as a chronic skin eruption characteristically reddish in colour, scaling and marginated, or as a systemic disease which involves not only the skin but also the heart, kidneys, gastrointestinal tract, lymph nodes, spleen, bone marrow, and the serous and synovial membranes. The systemic disease may arise de novo, as is most commonly the case, or may evolve from a pre-existing chronic form. Its course is characteristically severe, and the termination often fatal. Even in sub-acute cases, with prolonged periods of remission, the ultimate prognosis is grave.

The presence of numerous L.E. cells in heparinized aspirates of bone marrow is strongly suggestive of systemic lupus erythematosus, but these cells may also be present in small numbers in other collagen diseases. The ingested material in L.E. granulocytes consists partially of depolymerized desoxyribose-nucleic acid, and the stimulus to phagocytic activity appears to be a factor linked to the plasma gamma-globulin. The presence of this factor can be readily demonstrated in systemic lupus by the method of Mathis (1951), but has not been found in other conditions including dermatomyositis and discoid lupus.

Haematoxophil bodies, also containing depolymerized nucleic acid, are widely distributed in the tissues of patients with systemic lupus erythematosus, and, in the view of Klemperer (1952), are pathognomonic of the disease. In one series they were present in 32 of 35 cases (Klemperer and others, 1950), and in another (Gueft and Laufer, 1954) in thirteen out of fourteen cases, as compared with a control series of fifty cases, including eight instances of polyarteritis nodosa, from which they were absent. They are found most frequently in the kidneys, ovaries, lymph nodes, and heart.

Gueft and Laufer also stress the diagnostic significance of " wire-looping" and "hyaline thrombi" in the renal glomeruli, which they found in all their cases, and of " onion peel" periarterial fibrosis in the spleen, which was present in 60 per cent. 
Hypersensitivity is widely invoked as a possible cause of systemic lupus erythematosus, but it must be admitted that the fundamental nature of the disease is quite unknown. Gueft and Laufer have recently suggested that the fibrinoid substance does not result from an alteration in the collagen fibres and connective tissue ground substance but is a deposition of degraded nucleo-protein from the blood stream. Consideration of these facts recalls the speculation proffered by Robb-Smith (1952) that systemic lupus erythematosus may be a primary disorder of nucleo-protein metabolism, and that the L.E. plasma factor is possibly an enzyme which induces nucleo-protein denaturation.

The investigation of these two cases did not reveal any new or unusual features. Leucopenia and hypochromic anaemia, noted in both cases, are salient clinical features of the systemic disease, in which there not infrequently arises a terminal intercurrent infection, as exemplified by the pyaemia which developed in Case 2. In neither case was there clinical evidence of hypertension. Both cortisone and ACTH have proved disappointing therapeutically, and general clinical experience suggests that their effect is limited to the production of periods of remission.

Retinal changes not infrequently occur in systemic lupus erythematosus. Gold and Gowing (1953) described blurred discs, central exudate, and venous congestion in three of their 26 cases, of which 24 were females. Higher rates of ocular affection have been reported by Baehr and others (1935), who found some retinal change in 50 per cent. of their cases. Maumenee (1940) described retinal cytoid bodies, superficial retinal haemorrhages, and mild choroiditis in five cases of acute systemic lupus erythematosus. In all his cases, as in one of those reviewed above, profound toxaemia and cytoid body formation were prominent terminal events. These retinal lesions may wax and wane with exacerbations and remissions in the course of the illness and thus serve as an index of the degree of the severity of the systemic process. The micro-aneurysms present in Case 1 were probably the result of the diabetes from which the patient had suffered for 8 years.

In sections stained with haematoxylin and eosin, cytoid bodies appear as spherical or pyriform eosinophilic structures with a deep red core and an average diameter of $25 \mu$ (Fig. 4 , overleaf); aggregations of less than a dozen or of many scores are visible ophthalmoscopically as superficial fluffy exudates (cotton wool spots) located in the nerve fibre layer of the retina. It has not, however, been shown that all fluffy exudates consist of cytoid bodies.

By examining cytoid bodies dissected from fresh, unfixed retinae, Kiewe and Hart (1951) demonstrated that they may be fusiform or even tubular in outline with filiform terminal processes. They also showed that the bodies have a high mucopolysaccharide content, and do not stain with specific nuclear stains, and that in flat preparations with injected vessels the neighbouring retina is almost devoid of patent capillaries. 


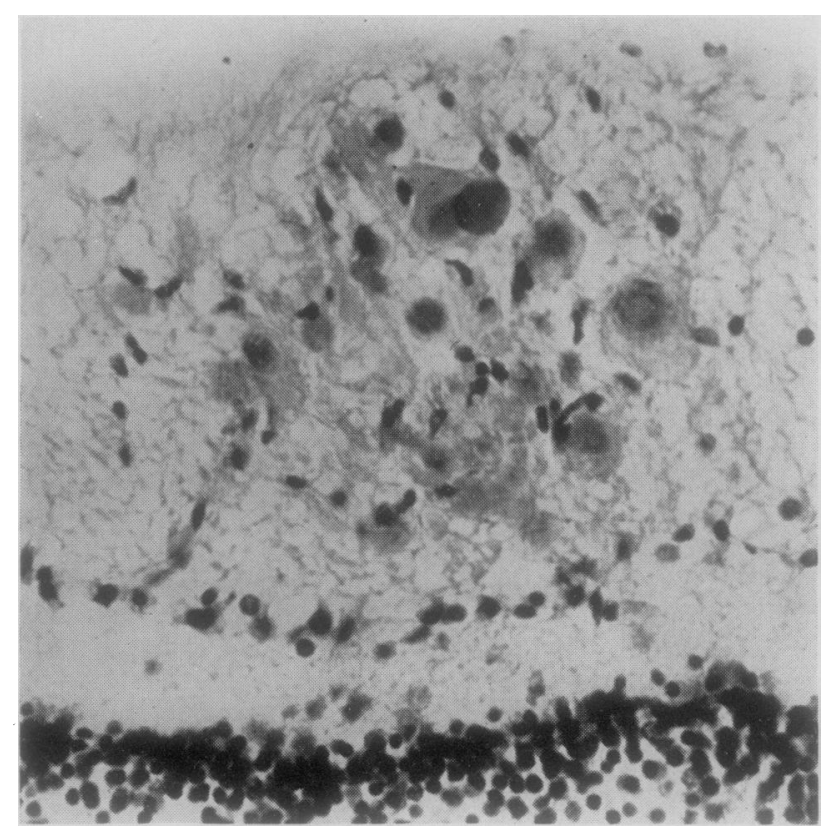

Fig. 4.-Photomicrograph of cytoid bodies, clearly showing more deeply staining core. Haematoxylin and eosin. $\times 500$.

Retinal cytoid bodies occur in hypertensive retinopathy and in diabetic retinopathy accompanied by hypertension. They are also seen in association with papilloedema, optic neuritis, and lacerations of the retina; in acute systemic lupus erythematosus, dermatomyositis, and scleroderma; in septicaemia, bacterial endocarditis, leukaemia, severe anaemia, and carcinomatosis. They have been reported by Friedenwald (1952) in polyarteritis nodosa and erythema multiforme, and we have observed them in an eye enucleated for thrombotic glaucoma; they do not, however, commonly occur in this condition and the possibility that the patient was hypertensive could not be excluded. The white retinal spots occurring in septic retinitis and first described by Roth (1872) are probably foci of cytoid bodies. Verhoeff (1921) found identical bodies in gliomata of the optic nerve and brain, and somewhat similar structures, usually, however, lacking the characteristic central core, have been demonstrated in many extra-ocular tissues by McLean (1935).

Cytoid bodies have been thought to be nerve fibres swollen by immersion in oedema fluid. In favour of this view is the occasional observation of nerve fibres in continuity with a cytoid body, and the fact that they are invariably located in the nerve fibre layer. Maumenee (unpublished work) has, however, demonstrated in flat preparations that cytoid bodies lie between the nerve fibres which pass round them without interruption. In addition, it is 
extremely unlikely that the very slender retinal nerve axons could swell to the relatively enormous diameter of $25 \mu$ or more without disrupting. Finally, while the retinal oedema is commonly diffuse, cytoid bodies occur in discrete aggregations separated by apparently normal nerve fibres.

Friedenwald (1948) demonstrated that cytoid bodies are regularly located between the terminal bifurcations of arterioles and concluded that they were degenerate glial cells in areas of ischaemic infarction. In most instances, however, he was unable to demonstrate arteriolar occlusion and presumed that such infarcts resulted from arteriolar spasm. This theory does not account for the fact that while glial cells pervade the retina cytoid bodies are invariably confined to the nerve fibre layer. Moreover, the number of cytoid bodies in a large focus, as for example Case 2 (Fig. 3), would seem to be greatly in excess of the number of glial cells normally present. Glial stains applied to cytoid bodies have proved somewhat inconclusive and do not indicate a glial origin with any certainty.

Verhoeff (1935) expressed the opinion that extravasated blood may, on occasions, be converted to cytoid bodies, and Kiewe and Hart, as a result of their extensive investigation, concluded that cytoid bodies were probably coagulated albuminous exudates moulded by the glial framework in which they lie. The darker pseudo-nucleus has not so far been satisfactorily accounted for, but may be due to inspissation in the centre of the body.

Whatever the precise nature of cytoid bodies may be, it is reasonably certain that the cardinal factor in their pathogenesis is damage to the endothelium of the capillaries, which increases permeability and permits the escape of plasma and red cells into the nerve fibre layer. Such capillary injury may result from circulating toxins, anoxaemia, or stagnation in the capillary bed due to obstruction or occlusion of the arterial supply or the venous outflow or from a combination of such factors. Regarded in this way cytoid bodies appear as exudates peculiar to the nerve fibre layer, but comparable in origin to other retinal exudates.

\section{Summary}

(1) The clinical features, course, and post-mortem findings are recorded in two cases of acute systemic lupus erythematosus.

(2) The accompanying fundus lesions are described.

(3) Histological changes in the retina and choroid are detailed and the pathogenesis of retinal cytoid bodies is discussed.

(4) General aspects of the disease are considered.

(5) The effect of treatment by ACTH is recorded in one case.

We wish to express our thanks to Dr. C. D. Evans and Dr. A. M. G. Campbell, of Bristol Royal Hospital, for permission to publish these cases, and for kindly affording access to their records. Our thanks are also due to Dr. A. L. Taylor, Consulting Pathologist, Bristol Royal Hospital, and to Dr. O. C. Lloyd, Department of Pathology, University of Bristol, who performed the autopsies and histological examinations. We are indebted to Dr. P. Hansell for the photomicrographs. 


\section{REFERENCES}

Baehr, G., Klemperer, P., and Schifrin, A. (1935). Trans. Ass. Amer. Phys., 50, 139.

Friedenwald, J. S. (1948). Trans. Amer. Acad. Ophthal. Otolaryng., 53, 73.

Wilder, H. C., Maumenee, A. E., Sanders, T. E., Keyes, J. E. L., Hogan, M. J., OWens, W. C., and Owens, E. U. (1952). "Ophthalmic Pathology", p. 319. Publ. by Amer. Acad. Ophthal. Otolaryng. and Armed Forces Institute of Pathology. Saunders, Philadelphia,

Gold, S. C., and Gowing, N. F. C. (1953). Quart. J. Med., 22, 457.

Gueft, B., and Laufer, A. (1954). Arch. Path. (Chicago), 57, 201.

KieWe, P. L., and Hart, F. Dudley (1951). Trans. ophthal. Soc. U.K., 71, 309.

Klemperer, P., Gueft, B., Lee, S. L., Leuchtenberger, C., and Pollister, A. W. (1950). Arch. Path. (Chicago), 49, 503.

(1952). "Progress in Fundamental Medicine”, ed. J. F. A. McManus, p. 51. Kimpton, London; Lea and Febiger, Philadelphia.

McLean, A. J. (1935). Arch. Ophthal. (Chicago), 13, 391.

Mathis, H. B. (1951). Blood, 6, 470.

Maumenee, A. E. (1940). Amer. J. Ophthal., 23, 971.

Unpublished work quoted by Friedenwald (1952).

Robb-Smith, A. H. T. (1952). Proc. roy. Soc. Med., 45, 811.

RoTH, M. (1872). Virchows Arch. path. Anat., 55, 197.

SEMON, H. C., and WolfF, E. (1933). Proc. roy. Soc. Med., 27, 153.

Verhoeff, F. H. (1921). Trans. Sect. Ophthal. Amer. med. Ass., 146. (1935). Arch. Ophthal. (Chicago), 13, 1082. 